\title{
EXPERIÊNCIA DOS FAMILIARES NO CONVÍVIO DE CRIANÇAS COMTRANSTORNO DO ESPECTRO AUTISTA (TEA)
}

Rafaela da Rosa Hofzmann¹,Márcia Perondi', Jouhanna Menegaz², Soraia Geraldo Rozza Lopes', Dayanne da Silva Borges ${ }^{1}$

Resumo: Objetivo: conhecer a experiência dos familiares no convívio de crianças com TEA. Metodologia: pesquisa qualitativa, realizada através de Grupo Focal com familiares de oito crianças com diagnóstico de TEA. A coleta de dados ocorreu no mês de setembro de 2016, através de questões norteadoras discutidas no grupo. Os relatos foram gravados em áudios e transcrito pelos autores. A análise foi realizada a partir da Análise de Conteúdo. Resultados: A partir da análise dos dados surgiram três categorias: 'a descoberta do autismo'; 'experiências dos familiares após o diagnóstico de autismo' e 'atendimento em saúde da criança com autismo'. Conclusão: o autismo é um transtorno que causa muitas adaptações e mudanças na vida dos familiares envolvidos, surgindo a necessidade do apoio dos profissionais de saúde no suporte dos cuidados prestado a estas crianças.

Descritores: Autismo; Relações Familiares; Acontecimentos que Mudam a Vida; Enfermagem.

\section{THE EXPERIENCE OF FAMILIARS IN THE COEXISTENCE OF CHILDREN WITH AUTISTIC SPECTRUM DISORDER (ASD)}

Abstract: Objective: to know the experience of family members living with children with ASD. Methodology: qualitative research, carried out through a Focal Group with relatives of eight children diagnosed with ASD. Data collection occurred in September 2016, through guiding questions discussed in the group. The reports were recorded in audios and transcribed by the authors. The analysis was made from the Content Analysis. Results: From the analysis of the data emerged three categories: 'the discovery of autism'; 'Family experiences after the diagnosis of autism' and 'health care of the child with autism'. Conclusion: autism is a disorder that causes many adaptations and changes in the lives of the family members involved, resulting in the need of the support of the health professionals in the support of the care provided to these children.

Descriptors: Autism; Family Relations; Life Changing Events; Nursing.

\section{LA EXPERIENCIA DE LOS FAMILIARES EN LA CONVIVENCIA DE NIÑOS CON TRANSTORNO DEL ESPECTRO AUTISTA (TEA)}

Resumen: Objetivo: conocer la experiencia de los familiares en la convivencia de niños con TEA. Metodología: investigación cualitativa, realizada a través de Grupo Focal con familiares de ocho niños con diagnóstico de TEA. La recolección de datos ocurrió en el mes de septiembre de 2016, a través de cuestiones orientadoras discutidas en el grupo. Los relatos fueron grabados en audios y transcrito por los autores. El análisis fue realizado a partir de la Análisis de Contenido. Resultados: A partir del análisis de los datos surgieron tres categorias: 'el descubrimiento del autismo'; “Experiencias de los familiares después del diagnóstico de autismo" y "atención en salud del niño con autismo". Conclusión: el autismo es un trastorno que causa muchas adaptaciones y cambios en la vida de los familiares involucrados, surgiendo a la necesidad del apoyo de los profesionales de salud en el soporte de los cuidados prestados a estos niños.

Descriptores: Autismo; Relaciones Familiares; Acontecimientos que cambian la vida; Enfermería. 


\section{INTRODUÇÃO}

O Autismo é classificado no grupo de Transtornos do Espectro Autista (TEA). Com base no DSM 5, sabe-se que o diagnóstico é clínico, feito a partir da observação da criança, relato dos cuidadores eaplicação de instrumentos específicos. A causa do autismo ainda é desconhecida, considerando-se o envolvimento de fatores genéticos, idade avançada dos pais, baixo peso ao nascer e exposição fetal ao ácido valpróico(1).

Associado ao diagnóstico de TEA, estão as manifestações comportamentais acompanhadas de déficits de comunicação e interação social, comportamentos repetitivos e estereotipados, havendo assim alterações precoces nas áreas de socialização, comunicação e cognição, com variações individuais. Os comprometimentos advindos do autismo trazem impactos ao autista e sua família, que devem se ajustar às novas demandas e às exigências advindas da condição de deficiência da criança ${ }^{(2,3)}$

Esse processo de adaptação da família pode gerar efeitos estressores, com prejuízo às atividades sociais, expondo as famílias a cuidados extensos e por longos períodos de dedicação à criança com TEA. Durante o trajeto de adaptação dessas familias, há sentimentos de desânimo, desesperança e cansaço, que podem prejudicar a estrutura familiar, fundamental para o progresso da criança autista(4).

Ainda são escassas as pesquisas em torno do autismo, assim como a atenção e cuidado direcionado aos familiares dessas crianças, considerando que o transtorno exige muitas adaptações e mudanças nas vidas dos cuidadores e a deficiência no suporte oferecido a estes. Diante do exposto, questiona-se: Qual é a experiência dos familiares no convívio de crianças com TEA?

Logo, este manuscrito objetiva conhecer a experiência dos familiares no convívio de crianças com TEA. Justifica-se sua relevância para demonstrar a importância da detecção precoce, bem como o acolhimento às famílias de autistas, buscando, na Atenção Primária em Saúde e na atuação do profissional enfermeiro, a porta de entrada para esse acolhimento e atenção, dispensados aos familiares em todo processo que envolve o autismo.

\section{METODOLOGIA}

\section{Tipo de estudo}

Trata-se de um estudo de natureza qualitativa com abordagem exploratória. A pesquisa qualitativa busca explorar os fenômenos em sua essência ou a experiência vivida.

\section{Participantes da pesquisa}

Os participantes foram familiares de crianças com TEA que utilizam os serviços de Estimulação Essencial(5).

Os critérios de inclusão foram: ser familiar da criança com diagnóstico de TEA, que utilize os serviços de estimulação essencial da APAE.

\section{Local do estudo}

O local do estudo foi a Associação de Pais e Amigos dos Excepcionais (APAE), de um município da Grande Florianópolis, Estado de Santa Catarina, cuja coleta de dados ocorreu através de Grupo Focal.

\section{Coleta de dados}

O grupo focal foi realizado no dia 22 de setembro de 2016 conforme informado no convite realizado via telefônica e por e-mail. Previamente ao início da sessão, os participantes receberam esclarecimentos acerca da pesquisa, assim como da metodologia de gravação e filmagem que seriam empregadas durante a sessão e do sigilo ao material produzido.

A fim de estimular a discussão do tema entre os participantes, a sessão teve início com a leitura de uma vinheta fictícia, criada pelos autores, acerca de uma família com uma criança autista ${ }^{(6)}$. A sessão foi conduzida, abordando categorias e questionamentos pertinentes, em que cada participante pode expressar suas vivências a partir dos questionamentos apontados.

\section{Análise dos dados}

Os registros dos dados obtidos na sessão de grupo focal foram ordenados ${ }^{(6)}$, classificados e analisados através de análise de conteúdo conforme as premissas de Minayo(7). Para a ordenação dos dados foram realizadas: (a) transcrição das falas do Grupo Focal (gravação e filmagem); (b) releitura do material; (c) organização dos relatos, iniciando uma classificação.

\section{Aspectos Éticos}

Os procedimentos éticos do estudo são registrados através da anuência do Comitê de Ética da Universidade Paulista, através do parecer no 1.730.878. Foi realizada a leitura do Termo de Consentimento Livre e Esclarecido (TCLE) para os participantes, com esclarecimentos de eventuais dúvidas e coleta das assinaturas, como expressão do consentimento de participação na pesquisa.

\section{RESULTADOS}

Foram identificados e convidados a participar da pesquisa familiares de 13 crianças que cumpriam os critérios de inclusão. Obteve-se aceite informal (pessoalmente ou via ligação telefônica) de $100 \%$ dos indivíduos convidados, contudo apenas oito familiares compareceram à data do encontro. Considerando que a técnica metodológica do 
Grupo Focal permite a média de seis a onze participantes, o número alcançado compôs o grupo focal, realizado em uma única sessão.

A amostra foi composta por avós, pais e mães de crianças com autismo, com faixa etária de 21 a 66 anos de idade, grau de escolaridade majoritariamente de nivel fundamental incompleto, como principal ocupação "Do Lar", e renda mensal entre $\mathrm{R} \$ 480,00$ a 3.500,00.

A partir da análise dos dados surgiram três categorias: a descoberta do autismo por parte das famílias; as experiências dos familiares após o diagnóstico do autismo; e o atendimento em saúde da criança com autismo.

\section{A descoberta do autismo}

Nessa categoria, foram agrupados os relatos dos familiares desde o nascimento até os dois anos e sete meses de idade da criança com TEA. As observações relatadas foram, principalmente, a atitude de "organizar" e "girar" objetos. Adicionalmente, foram percebidos movimentos repetitivos do próprio corpo, como "andar na ponta dos pés", "sacudir as mãos" e "mexer o tronco para frente e para trás", assim como ausência ou parada repentina de fala, ausência de resposta ou contato visual, isolamento, choro intenso ao mudar de ambiente, agressividade, insônia, rejeição ao colo ou contato físico: “Tudo que ele pegava ele organizava, ele não brincava. Era meia, era sapato das crianças, ele se isolava num cantinho e ficava organizando" (vermelho); "Quando eu vi ele não falava, começou a travar, não escutava, não respondia" (verde).

Quanto às atitudes tomadas frente à percepção das diferenças, em sua maioria, houve a procura por profissional fonoaudiólogo, seguido de médico pediatra, neurologista, otorrinolaringologista e a Fundação Catarinense de Educação Especial (FCEE). Na grande maioria, os familiares não tinham o conhecimento do que era o autismo: "Achava que era um retardo mental. [...] Não sei, não conhecia, não sabia" (verde);

\section{Experiências dos familiares após o diagnóstico do autismo}

Os familiares apresentaram reações diversas, como aceitação, preocupação, sofrimento, negação, sensação de impotência e previsão de momentos turbulentos.

Deacordo com os relatos, a rotinafamiliarsofreu profundas mudanças devido ao cuidado e atenção dispensados à criança, dentre elas a necessidade de renunciar às atividades profissionais: "[...] Minha vida é só ele! É em função dele!" (preto).

Em relação às dificuldades enfrentadas, o comprometimento financeiro foi citado como maior problema. O orçamento familiar passou a ser comprometido com gastos em tratamentos necessários à criança, somados à perda de emprego de alguém da casa: "Mudou, fui dispensada do serviço, não consegui mais serviço. Sempre tem que andar com ele pra cima e pra baixo, só eu! [...]" (verde).

Em contraste com as dificuldades, os familiares relataram a aprendizagem que tiveram a partir da convivência com a criança autista e os pontos positivos existentes nesta convivência. Dentre os relatos, alguns enfatizaram a valorização das pequenas vitórias relacionadas à evolução no desenvolvimento da criança autista, situações antes despercebidas pelos mesmos: "Mas o que a gente aprende, é basicamente comemorar com as pequenas vitórias [...]" (laranja).

Foram relatadas também habilidades e/ ou facilidades que a criança apresenta em sua vivência cotidiana, na perspectiva dos familiares. Dentre essas, as habilidades/ facilidades com música, aparelhos smartphones, letras e números, e a compreensão de vídeos e músicas em inglês: "A minha, por exemplo, tudo que tem letras e números atrai ela [...]" (laranja); "[...] Ele estava vendo um desenho em inglês, e o desenho falava: Triangle! Ele pegava o triângulo e colocava lá" (vermelho)

Quanto à perspectiva de futuro apontado pelos familiares em relação à criança com autismo, detectou-se a busca pelo melhor apoio e amparo hoje, evitando dificuldades nos dias vindouros, além de não dispensar a fé em Deus e a esperança em vitórias e conquistas: "O futuro da minha filha, enquanto eu tiver vivo, eu quero fazer o melhor por ela. Aí depois eu não sei né! Espero em Deus!" (azul).

\section{Atendimento em saúde da criança com autismo}

Os relatos demonstram que a Unidade Básica de Saúde não tem uma participação efetiva no atendimento e acompanhamento da criança autista. Isto decorre da demora no agendamento das consultas e exames pelo Sistema Único de Saúde (SUS), fazendo com que os familiares recorram a outras vias de atendimento. Adicionalmente, os familiares relataram a inexistência da atuação do profissional enfermeiro em qualquer momento do processo da doença, seja antes ou após o diagnóstico: "[...] Eu acho que o profissional de enfermagem tem que saber sobre isso, porque lida muito com criança. E o quanto antes a gente poder ter um diagnóstico ou uma familia ser orientada, mais chances nós temos de ter crianças bem desenvolvidas!"(laranja).

Em relação à APAE, os familiares afirmaram que a criança utiliza os serviços de estimulação essencial da instituição semanalmente. Porém, preferem levar a criança em atividades extras, fora da instituição, buscando reforçar o acompanhamento e desenvolvimento da criança.

\section{DISCUSSÃO}

Semelhante ao que se observa nesse estudo, a literatura 
afirma que os sintomas do TEA são reconhecidos entre os 12 e 24 meses de vida da criança ${ }^{(1)}$. Frente às percepções das diferenças comportamentais da criança autista, os familiares recorrem primeiramente ao profissional fonoaudiólogo, baseando-se na hipótese de distúrbios auditivos. Apesar da suspeita diagnóstica inicial, a surdez é geralmente descartada com o evoluir das investigações ${ }^{(1,8)}$

O desconhecimento ou ainda o conceito equivocado sobre o autismo, relatado pelos participantes da presente pesquisa, evidencia a importância da disseminação de informações sobre o TEA para a sociedade, sobretudo para população leiga, que pode vir a conviver diariamente e diretamente com o transtorno(9). Para que o autista seja acolhido, faz-se necessário que o conceito, as características e as formas de tratamento sejam conhecidos por todos, facilitando o acolhimento pela família, especialistas e sociedade, fazendo com que o individuo receba o apoio necessário para seu desenvolvimento cognitivo, pessoal e social.

Em relação às experiências na vida familiar após o diagnóstico de TEA, foram observados vários relatos, dentre eles, as mudanças cotidianas nas quais os familiares tiveram que estabelecer após o diagnóstico. Alguns estudos relatam que a família é colocada frente à sentimentos de luto, negação, tristeza e culpa, que associada a falta de conhecimento do transtorno, pode agravar a negação quanto ao diagnóstico (10,11)

Quanto às dificuldades advindas com o diagnóstico de autismo, destaca-se a dificuldade financeira, seguida de relatos de dificuldade em frequentar ambientes diferentes ao habitual da criança e a aceitação das demais pessoas. A literatura cita que, principalmente, a figura materna reconhece no filho autista a dificuldade em relacionar-se, inflexibilidade ao barulho, falta de vínculo e dificuldades de comunicação. Tal situação faz com que a família tenha que se adaptar às exigências que a perturbação do transtorno do espectro autista traz, exigindo atenção permanente dos cuidadores, culminando no afastamento do convívio social(12).

Adicionalmente, evidenciam-se pontos considerados positivos pelos familiares, em relação à aprendizagem adquirida na convivência com a criança autista. Segundo a literatura, a afetividade é de extrema importância para os autistas, pois proporciona aproximação e a criação de vínculo com os familiares, subsidiando o bom desenvolvimento da criança e fortalecendo a saúde emocional de toda a família(13).

Estudos indicam a alta capacidade de memória em crianças autistas, observada pelo aprendizado mecânico, cuja tarefa realizada não requer a compreensão do contexto global. Autistas possuem "ilhotas de habilidades especiais", áreas com alta habilidade, desenvolvendo interesses específicos, associados a uma excelente memória visual ${ }^{(14)}$
Em relação às perspectivas futuras, observa-se, entre os participantes deste pesquisa, assim como na literatura(15), preocupações relacionadas ao futuro dos indivíduos com diagnóstico de TEA, principalmente no que se refere a sua independência, advindo o desejo da inserção na sociedade pela busca da "normalidade". Os estudos apontam que a intervenção precoce é fator fundamental para ganhos com relação ao quadro clínico do autismo, adquirindo ganhos significativos e duradouros no desenvolvimento da criança(16).

No que diz respeito ao atendimento de saúde à criança autista, foi observada a falta da participação efetiva da Atenção Básica na vida das famílias e das crianças autistas, bem como a ausência do profissional enfermeiro antes e/ ou após o diagnóstico de autismo. Visto que a Atenção Básica deve realizar ações de promoção, prevenção e reabilitação em saúde, dentre elas, deve estar a consulta de enfermagem, quando é possível identificar sinais de atraso no desenvolvimento infantil. O enfermeiro também tem seu papel de educador na medida em que procura orientar as famílias sobre a patologia e os sinais de atraso no desenvolvimento. A enfermagem ainda aparece de forma muito tímida no atendimento e tratamento de autistas, em parte, devendo-se à falta de conhecimento da patologia pelos profissionais(17)

Os atendimentos semanais da criança autista relacionados à sua estimulação, tiveram seu foco voltado para a APAE. O brincar é um recurso terapêutico utilizado na estimulação precoce, visto que a brincadeira estimula o desenvolvimento das crianças durante a infância, quando evidencia o significado dos objetos, o aprendizado da linguagem e realidade da vida(18).

Essa terapêutica está fundamentada na plasticidade cerebral, ou seja, na habilidade do sistema nervoso de se remodelar em função das experiências do indivíduo, reformulando suas conexões em função das necessidades e dos fatores do meio ambiente. A importância da estimulação precoce em autistas justifica-se pelo presente fator, visto que certas regiões encefálicas podem substituir as funções afetadas, conquistando novas áreas de desenvolvimento ao autista ${ }^{(19)}$. Outro fator importante de ser destacado, é que a inclusão da criança com deficiência está articulada ao conhecimento dos professores na área e na necessidade de capacitação para o trabalho. Para que haja inclusão, os professores necessitam ampliar seu leque de conhecimentos, investir na sua formação continuada, para aprender $e$ desenvolver as potencialidades dessas crianças $^{(3)}$.

\section{Limitações do estudo}

O presente estudo apresentou algumas limitações, principalmente no que se refere à abrangência, estando restrito a um único local; a limitação temporal, visto que 
a coleta de dados foi realizada em um único encontro e a limitação relacionada ao número reduzido de participantes.

\section{Contribuição do estudo para a prática}

O presente estudo aponta a tamanha importância que o conhecimento traz para a vida dos familiares e o bom prognóstico do autista. A informação precoce do que se trata o autismo e quais os sinais e sintomas tornariam o diagnóstico ainda mais precoce, potencializando o desenvolvimento da criança e tornando mais fácil a aceitação e entendimento para os familiares.

Percebe-se a necessidade da maior participação da Unidade Básica de Saúde na vida das crianças e dos familiares, visto que, é através do acompanhamento do desenvolvimento da criança na primeira infância que é observada os primeiros sinais de alteração em seu desenvolvimento. Evidenciamos o papel fundamental do profissional enfermeiro frente à consulta de enfermagem e no acolhimento à criança e sua família.

\section{CONCLUSÃO}

Tendo em vista o objetivo de conhecer a experiência dos familiares no convívio com crianças com TEA, conclui-se que o transtorno promove muitas adaptações e mudanças na vida dos familiares envolvidos, e que estes recebem pouco suporte para se adequarem a essa situação. Adicionalmente, faz-se necessária a inclusão de políticas públicas que promovam a capacitação dos profissionais da rede, para a detecção e acolhimento da família de autistas, assim como, para que o profissional enfermeiro seja coautor dessa detecção precoce.

Contudo, diante da pouca exploração do conhecimento nessa temática, fica evidente a necessidade da realização de mais estudos acerca da atenção voltada à família da criança autista, bem como do papel do profissional enfermeiro frente ao diagnóstico, assim como na identificação dos primeiros sinais e sintomas do autismo na Atenção Primária em Saúde.

Ao final desse trabalho, consegue-se perceber o quão impactante é o diagnóstico de TEA para a família. A falta de conhecimento sobre o assunto impera sobre a maioria, trazendo insegurança e sentimentos diversos. Ficou claro que, o diagnóstico precoce trona-se a chave para o bom prognóstico e desenvolvimento da criança autista, que quanto mais jovem maior as chances de melhora do quadro devido à plasticidade cerebral.

As famílias atravessam momentos de tristeza, luto, medo diante de uma situação nova e sem respostas para tais. Muitos dedicam-se integralmente a criança autista, devido a grande quantia de terapias necessárias. Contudo, percebese que em muitos casos mesmo em meio à desesperança e o medo, os familiares conseguem olhar pelo lado positivo, acreditando sempre no potencial da criança, tendo fé no futuro, e enxergando pontos positivos, vistos como especiais por eles, com relação ao autista.

Perante o estudo, foi possivel verificar a falta de assistência prestada pelo SUS e do profissional enfermeiro. Onde há longa espera por consultas com especialistas, e falta de informação e participação ativa do enfermeiro no que tange o desenvolvimento infantil e o acolhimento a família.

Contudo, tem-se a certeza de que ainda há muito a se fazer quando se trata de autismo. E que a informação é a grande arma para o diagnóstico precoce e o bom prognóstico da criança e de sua família.

\section{Contribuições dos autores}

Rafaela da Rosa Hofzman, Marcia Perondi, Jouhanna Menegaz e Soraia Geraldo Rozza: concepção e desenho do estudo; Rafaela da Rosa Hofzman, Marcia Perondi e Dayanne da Silva Borges: coleta e organização dos dados; Rafaela da Rosa Hofzman, Marcia Perondi, Jouhanna Menegaz, Soraia Geraldo Rozza e Dayanne da Silva Borges: análise e interpretação dos dados e redação do artigo; Jouhanna Menegaz e Soraia Geraldo Rozza: revisão crítica do artigo; Rafaela da Rosa Hofzman, Soraia Geraldo Rozza e Dayanne da Silva Borges: revisão final do artigo. 


\section{REFERÊNCIAS}

1. American PsychiatricAssociation. Manual Diagnóstico e Estatístico de Transtornos Mentais - DSM 5. 5. ed. Porto Alegre: Artmed; 2014.

2. Moraes MM, Simões TM. Familiares de crianças e adolescentes com autismo: percepções do contexto escolar. Revista Educação Especial [Internet]. 2015 [cited 201820 mar]; 28 (52) 429-441. Available from: https://periodicos.ufsm.br/educacaoespecial/ article/view/14708

3. Bento TS, Castilhos GIC, Schoeller SD, Rocha PK, Toll AD, Soares MZ. Desafios para inclusão da criança com deficiência na escola. Enferm Foco 2015 [cited 20199 fev]; 6 (1/4), 36-40. Available from: http://revista.cofen.gov.br/index.php/enfermagem/ article/viewFile/574/256

4. Givigi RCN, Souza TA, Silva RS, Dourado SSF, Alcântara JN, Lima MVA. Implicações de um diagnóstico: o que sentem as familias dos sujeitos com deficiência?. Rev Dist da Com [Internet]. 2015[cited 201720 set]; 27 (3) 445-453. Available from: <https:// revistas.pucsp.br/index.php/dic/article/view/20892>

5. Associação de Pais e Amigos dos Excepcionais. Associação de Pais e Amigos dos Excepcionais - APAE de Palhoça/SC. Palhoça, SC. 2016 [acesso em 04 jun. 2016]. Available from: http://palhoca. apaebrasil.org.br/

6. Silva JRS; Assis SMB. Grupo focal e análise de conteúdo como estratégia metodológica clínica-qualitativa em pesquisas nos distúrbios do desenvolvimento. Cadernos de Pós-Graduação em Distúrbios do Desenvolvimento, 2018 [cited 201820 mar]; 10(1) 146-152. Available from: http://www.mackenzie.br/fileadmin/Graduacao/CCBS/PosGraduacao/Docs/Cadernos/cadernol0/62118_16.pdf

7. Minayo CSM. O Desafio do Conhecimento: pesquisa qualitativa em saúde. Rev Huc. 2014; 14, 261-360.

8. Freire MA, Pimenta RA, Pereira SM, Roesler H. Considerações sobre critérios diagnósticos de transtorno do espectro autista, e suas implicações no campo cientifico. Do corpo: ciências e artes [Internet]. 2018 [cited 201820 mar]; 7(1) 87-97. Available from: http://ucs.br/etc/revistas/index.php/docorpo/article/ view/5956

9. Santos JSO. Autismo e sociedade: definição, reflexão e relação social. Siqueira Campos. Monografia [Especialização em Genética] - Universidade Federal do Paraná. 2015 [cited 201606 nov]. Available from: http://acervodigital.ufpr.br/handle/1884/42363

10. Silva EM, Oliveira LA. Autismo: como os pais reagem frente a este diagnóstico? Unoesc \& Ciência - ACBS. 2017 Jan/ Jun;8(1),21-26.
11. Pereira ML, Bordini D, Zappitelli MC. Relato de Mães de Crianças com Transtorno do Espectro Autista em uma Abordagem Grupal. Cadernos Distúrbios. 2017 [cited 201807 fev];17(2),56-64. Available from: http://pepsic.bvsalud.org/scielo.php?script=sci_arttextSpid=S1519-03072017000200006

12. Pereira AIL. Sintomatologia psicopatológica, suporte social, preocupações parentais e percepção das relações fraternas em pais de crianças portadoras de perturbação do espectro do autismo. [Dissertação de Mestrado em Psicologia Clinica] Universidade de Trás-os-Montes e Alto Douro; 2016 [cited 2018 07 fev]. Available from: http://repositorio.utad.pt/bitstream/10348/7468/1/msc_ailpereira.pdf

13. Nascimento EP. Autismo e afetividade: implicações da dinâmica familiar no desenvolvimento da criança autista. [Trabalho de Conclusão de Curso de Bacharelado em Psicopedagogia] - Universidade Federal da Paraiba; 2016. [cited 201815 fev]. Available from: http://rei.biblioteca.ufpb.br/jspui/bitstream/123456789/2672/1/EPN27102017.pdf

14. Varela B, Machado PGB. Uma Breve Introdução sobre Autismo. Cad. Da Esc. De Educ. e Human. 2016 [cited 201606 nov]:1(11),25-39. Available from: file:///E:/Usuario/Downloads/ VarelaMachado2016Autismo.pdf

15. Semensato MR, Bosa CA. Crenças Indicativas de Resiliência Parental no Contexto do Autismo. Psicologia: Teoria e Pesquisa[Internet]. 2017[cited 201820 mar]; 33, 416-432. Available from: https://dx.doi.org/10.1590/0102.3772e33416

16. Basso RZ, Backes B, Bosa, CA. Diagnóstico do autismo: relação entre fatores contextuais, familiares e da criança. Psicologia: teoria e prática [Internet]. 2017 [cited 201820 mar]; 19(1) 152163. Available from: http://pepsic.bvsalud.org/pdf/ptp/v19nl/ v19nla09.pdf

17. Moura CMAB. Rastreamento do transtorno do espectro do autismo na consulta de enfermagem com a aplicação do M-Chat. Porto Alegre. Dissertação [Mestrado Profissional em Enfermagem] - Universidade do Vale do Rio dos Sinos. 2016 [cited 201606 nov]. Available from: http://www.repositorio.jesuita.org.br/handle/UNISINOS/5204

18. Alves MAG, Alves MP. I brincar como intervenção pedagógica nos transtornos do espectro do autismo. Revista Práxis. 2016 [cited 201627 mar]; 8(1), 73-82. Available from: http://revistas.unifoa.edu.br/index.php/praxis/article/viewFile/785/631

19. Reis PCKR, Bosco SD. Um olhar para o autista: um relato de experiência. E-Tech: Tec p Comp Ind (Especial Educação).2014 [cited 201627 mar]; Available from: http://revista.ctai.senai.br/ index.php/edicao0l/article/view/431/372 